\title{
Evidence for Azimuthal Asymmetry in Be Star Winds
}

\author{
Geraldine J. Peters \\ Space Sciences Center, University of Southern California, University Park, \\ Los Angeles, CA 90089-1341
}

\begin{abstract}
Extensive IUE observations from 1987-96 have provided compelling evidence for azimuthal asymmetry in the winds of Be stars. Two identified types of wind/FUV flux behavior are briefly discussed. Interpretations based upon both nonradial pulsations and rotation of one or more active regions appear plausible.
\end{abstract}

\section{The IUE Campaigns}

During the lifetime of the IUE satellite we carried through 12 multiwavelength campaigns to investigate the wind behavior in Be stars that have shown periodic variability in their optical spectra and light. A typical campaign consisted of 24-72 hours of uninterrupted, repeated IUE observations that were supported by simultaneous ground-based photometry, high resolution spectroscopy, and polarimetry. Two principal objectives were to ascertain if the wind strength correlates with the continuum flux and if the optical light variability is a result of a modulation of the photospheric temperature. A list of the 15 program stars and some of their properties can be found in Peters (1998). Three classes of behavior were identified: 1) correlated cyclic variability in wind strength and FUV flux, 2) cyclic wind variability only, and 3) cyclic FUV flux variability only. The first two groups are discussed here.

\section{The NRP Case}

Five of the Be stars for which we achieved good simultaneous ground-based spectroscopy ( $\lambda$ Eri, $28 \mathrm{Cyg}, \eta$ Cen, $\zeta$ Tau, \& 2 Vul) displayed nonradial pulsations (NRP) with sectorial, $\ell=-m=2$ modes (Hahula \& Gies 1994) and the periods derived from the NRP analysis agreed well with those from the FUV flux/wind variations (cf. Peters 1998, Table 1). The optical line profile data confirm that in $28 \mathrm{Cyg}$ (B3 IVe) and $\eta$ Cen (B2 IVe) a hot crest of the $\ell=2$ NRP mode crossed our line-of-sight when the star was brightest in the FUV. The latter stars also showed the strongest correlation between the wind strength and the FUV flux. Typical wind/photospheric behavior for this class of periodic Be stars is shown in Fig. 1 where the temporal variations in the strengths of the C IV wind and photospheric C III $(\lambda 1247)$ and Si II 


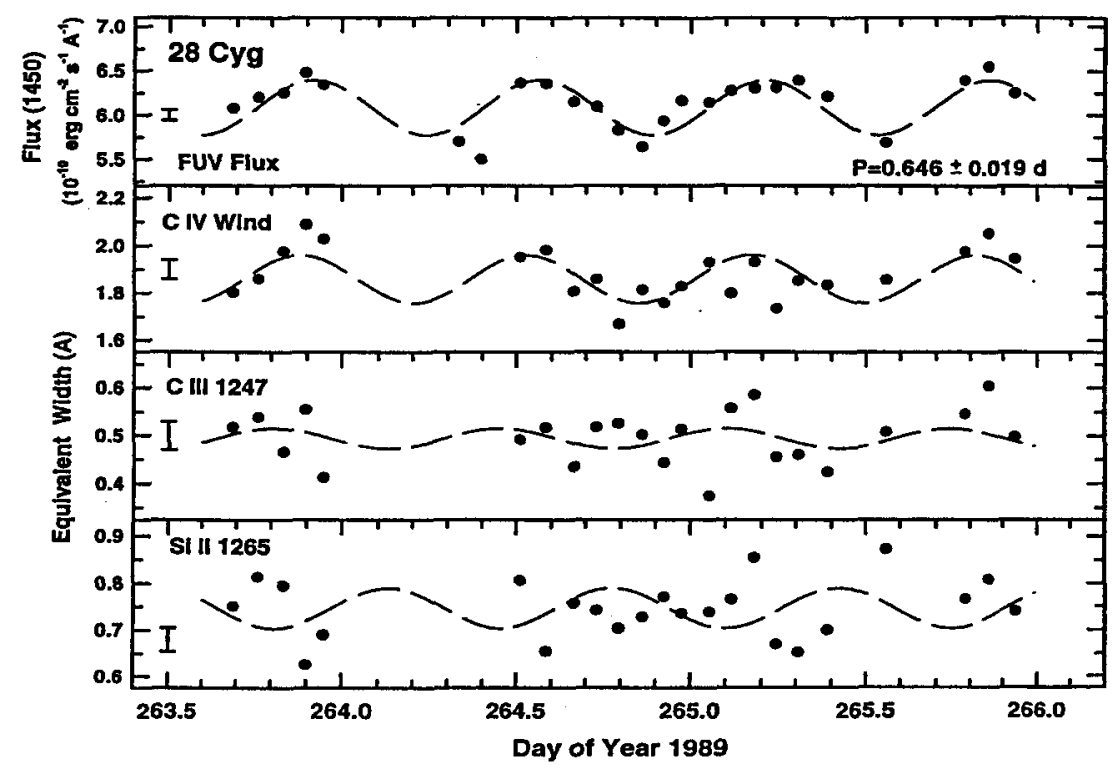

Fig. 1. The photometric, wind, and photospheric behavior of $28 \mathrm{Cyg}$ during the 1989 September campaign. The dashed lines are sine curve fits to the $1450 \AA$ flux and the EWs of the C IV wind and C III/Si II photospheric lines.

( $\lambda 1265)$ lines in $28 \mathrm{Cyg}$ are plotted and compared with the FUV flux. A sine curve fit to the FUV light variations revealed a $10 \%$ modulation at $1450 \AA$ with a period of $0 \mathrm{~d} 646$. The amplitude of the FUV light curve increases with decreasing wavelength, implying that the surface temperature is modulated by $500-750^{\circ}$. This interpretation is supported by the strength behavior of the temperature-sensitive photospheric $\mathrm{C}$ III and Si II lines (Fig. 1) that will be anticorrelated for early B stars (cf. results for $\eta$ Cen in Peters 1998). For $28 \mathrm{Cyg}$ and $\eta$ Cen there is compelling evidence that ongoing (retrograde) NRP produces a modulation of the surface temperature and that mass loss is enhanced over the hot crests.

\section{The Rotation Case}

For a few of the program stars ( $\omega$ Ori, $\psi$ Per, DU Eri, and EW Lac) cyclic variability in the wind was apparent but there did not appear to be a strong correlation with the FUV flux. These Be stars typically show no evidence of NRP or cyclic variations in their photospheric temperatures and the period for the modulation of their wind lines is comparable to what one would expect from stellar rotation alone. The periods seen in the stars undergoing NRP are shorter because the NRP motion is retrograde and for an $\ell=2$ 


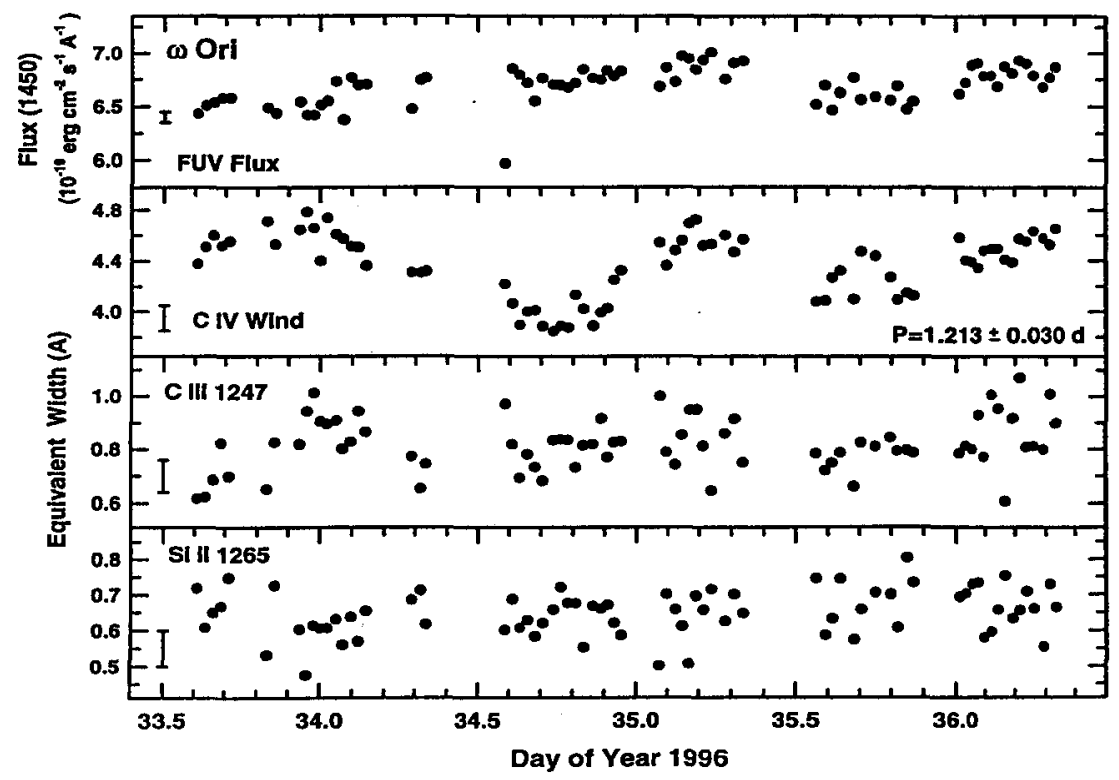

Fig. 2. Similar plot as in Fig. 1 for $\omega$ Ori. No variability was evident in the FUV flux or photospheric lines, but the wind was clearly modulated with a period of $\sim 1$. 2 that is comparable to the star's expected rotational period.

mode there are two crests/troughs that we see each rotational cycle. The temporal flux/wind/photospheric behavior observed in $\omega$ Ori (Fig. 2) can be compared with the NRP objects 28 Cyg (Fig. 1) and $\eta$ Cen (Peters 1998). The observations imply that enhanced mass loss occurs from an active region on the star that sweeps past our line-of-sight with each stellar rotation, but this region does not appear to differ substantially in temperature from the surrounding photosphere.

I extend my thanks to D. Gies, H. Henrichs, D. McDavid, and J. Percy who participated in most of the multiwavelength campaigns. This project was partially supported by NASA grants NSG-5422, NAG5-1296, \& NAG5-2313.

\section{References}

Hahula, M. E., Gies, D. R. (1994): in Pulsation, Rotation, and Mass Loss in EarlyType Stars, ed. L. A. Balona, H. F. Henrichs, J. M. LeContel, (Kluwer,Dordrecht, Boston, London), 100-101

Peters, G. J. (1998): in ESO Workshop on Cyclical Variability in Stellar Winds, ed. L.Kaper \& A. Fullerton (Berlin: Springer), 127-133 


\section{Discussion}

H. Lamers: In most stars where the equivalent width of $\mathrm{C}$ IV changes in phase with the FUV flux, you have direct evidence that the ionisation is due to photoionisation rather than to Auger-ionisation. Do you see that this correlation depends on spectral type? (You expect it to disappear in later B stiars.)

G. Peters: It has yet to be determined whether the modulation in the wind is entirely caused by the changing FUV flux due to the NRP. Detailed modelling will have to be undertaken to find out whether the wind is entirely radiatively driven or if the NRP imparts significant amounts of mechanical energy to assist the acceleration of the wind. There is no apparent correlation of the degree of FUV-wind variability with spectral type (nor $v \sin i$ ). The object with the earliest spectral type ( 2 Vul, B0.5 IVe) showed one of the weakest wind modulations; at the other extreme, our coolest star $\psi$ Per (B IIIe-shell) displayed the greatest wind variation.

R. Ignace: Can you comment on what the V/R ratio (e.g., in $\mathrm{H} \alpha$ ) does or is expected to do for your three classes of Be stars?

G. Peters: In the NRP case one sometimes observes $V / R$ variations in the $\mathrm{He} \mathrm{I}$ lines (also paralleled in $\mathrm{H} \alpha$ ) that have the same period, and are in phase with the photospheric NRP. This is the so-called "searchlight effect", where the enhanced FUV flux from the hot crest produces greater ionization in the portion of the disk just above the crest. V/R tends to be $\sim 1.0$ at NRP phases of 0.25 and 0.75 , when the hot/cool patches are aligned along our line-of-sight. A good example is $28 \mathrm{Cyg}$. In the other two cases one might see either short-term or long-term $\mathrm{V} / \mathrm{R}$ changes. Several of the Be-shell stars (e.g., $48 \mathrm{Lib}, \zeta \mathrm{Tau}$ ) display long-term $\mathrm{V} / \mathrm{R}$ variations in $\mathrm{H} \alpha$ that are likely due to precessing spiral density waves. I would think a fast-moving stream of material from an active region on a star (case 2) would make conditions favorable for generating such spiral waves.

P. Koubský: $\psi$ Per is known to be probably the strongest Be-radio star. Is it possible that there is a connection between the radio emission and the deviation of $\psi$ Per on the diagram you have just shown?

G. Peters: The radio emission would come from a large region of space; but if, as the UV data imply, there is an active region of mass loss in the photosphere (perhaps due to a magnetic field) a great deal of material could be built up at large distances from the star. 